Urologe 2014 · 53:1733-1734

DOI 10.1007/s00120-014-3707-x

Online publiziert: 14. November 2014

(c) Springer-Verlag Berlin Heidelberg 2014

\section{W. Bühmann}

Facharzt für Urologie - Andrologie, Medikamentöse Tumortherapie, Qualitätsmanagement, Sylt OT Keitum

\title{
Versorgungsforschung - schon wieder eine neue Sau im Dorf?
}

So, liebe Kolleginnen und Kollegen,

reagieren Sie häufig, wenn Sie - in welchem Zusammenhang auch immer mit diesem Thema in Kontakt kommen; gleichsam wie mit einem unbekannten Virus: ich kenne es nicht, aber wenn es mich erfasst, werde ich krank. Kein Wunder, hat doch die „Erfinderkaste“ dieses Gebietes schlichtweg vergessen, die $\mathrm{Zu}$ lieferer, Umsetzer, Anwender und Leidtragenden darüber zu informieren, dass das jetzt "angesagt“ und was es ist bzw. was es sein soll - bereits seit 15 Jahren! Nur eine Gruppe war von Beginn an klar definiert: die Nutznießer, sprich Kostenträger im Gesundheitswesen. Profan sedimentiert bedeutet Versorgungsforschung aus Sicht derer nichts anderes als ein in arztfremder Theoriesprache verkleidetes Instrument zur "Allokation" oder auch in Deutsch: „Kostendämpfung“, ein bewährtes, hochpotentes Allergen für alle Heiler und Helfer im Gesundheitswesen.

Mehrere Versuche, dieses Thema in der Urologie zu etablieren, scheiterten bisher an der übertheoretisierten, gestelzten Terminologie und fehlender (Be) Greifbarkeit - wir starten in diesem Heft einen neuen Versuch, uns dem friedlich zu nähern. Dazu zwei Zitate:

Während der letzten 15 Jahre hat sich die Versorgungsforschung auch in Deutschland etabliert. Anfangs häufig als vierte (neben der grundlagenorientierten, der krankheitsorientierten und der patientenorientierten Forschung) Säule der Gesundheitsforschung bezeichnet (Neugebauer, Pfaff, Schrappe \& Glaeske, 2008), hat sie als in- terdisziplinäres, eigenständiges Forschungsgebiet, das u. a. "die Kranken- und Gesundheitsversorgung und ihre Rahmenbedingungen beschreibt [..] und die Wirksamkeit von Versorgungsstrukturen und -prozessen unter Alltagsbedingungen evaluiert" (Pfaff, 2003)..."

„Die Versorgungsforschung untersucht die Strukturen und Prozesse unseres Gesundheitssystems unter Alltagsbedingungen. Forschungsgegenstand sind die Anwendung von Arznei-, Hilfs- und Heilmitteln, die Qualität der Versorgung mit diagnostischen und therapeutischen Methoden oder der Nutzen und die Risiken des Einsatzes von medizinischen Geräten. Die Versorgungsforschung dient der gesundheitspolitischen Steuerung und ermöglicht Entscheidungen über Versorgungsleistungen auf der Basis aktueller wissenschaftlicher Erkenntnisse."

Alles klar? Glaube ich nicht. Vielleicht hilft ein weiterer Übersetzungsversuch: „Was tun wir, was kostet es und was kommt dabei heraus?" Bisher hatten wir uns an randomisierte, placebokontrollierte, doppelblinde Studien (RCT, „randomised controlled trial“) gewöhnt, die unbeeinflusst von jeder Realität Arzneimittel, Methoden, Instrumente und Behandlungstechniken auf ihre Wirksamkeit hin evaluieren, weiterhin unzweifelhaft unverzichtbar für das Prinzip „primum nihil nocere“ - für die Sicherheit der uns anvertrauten Patienten.

Aber der Mensch, er ist nicht so - Begleiterkrankungen, individuelle Befindlichkeiten, genetische Dispositionen, unvorhersehbare Konstellationen und Wechselwirkungen in der komplexesten Sackgasse der Schöpfung pulverisieren häufig genug diese Erkenntnisse reiner Lehre und hehrer Wissenschaft.

Noch ein Versuch, gerne lasse ich mich für den unsensiblen Vergleich mit einer gänzlich unmenschlichen, aber so beliebten Maschine schelten: wenn Ferrari ein neues Formel-1-Auto mit der gesamten stupenden Ingenieurskunst und jahrzehntelangen Erfahrung der Mutter aller Rennställe konstruiert und damit das theoretisch schnellste, stabilste, sicherste und sparsamste Wunderwerk auf die Räder stellt, ist das eine RCT.

Setzt Sebastian Vettel sich hinter das Steuer und dreht ein paar Runden nur wenige Zehntelsekunden langsamer als geplant, weil die Reifen zu schnell verschleiBen, die Asphaltbeschaffenheit nicht passt, eine 2-Euro-Münze auf der Fahrbahn zum Abflug ins Kiesbett führt, der Sitz seinem Model-Glutaeus nicht schmeichelt und „der Bock einfach nichts für mich ist", dann ist das Versorgungsforschung oder auch: Realität. Wenn er dann noch das Budget sprengt, gehört das Wunder noch vor Ende des ersten Rennens in die Wertstofftonne.

Was ist bisher schief gelaufen? Nicht die Kostenträger dürfen spargeile, menschenfeindliche Profiteure der Versorgungsforschung sein - Ärztinnen und Ärzte als alleinige Siegelbewahrer der im Rahmen der Begleitung ihrer Patienten erhobenen Daten sollten diese vertraulich auswerten, um daraus abzuleiten, was

Dr. W. Bühmann ist BDU-Pressesprecher. 
die "Gesundheitstreuhänder" von dem den Versicherten ohne Einflussmöglichkeit enteigneten Vermögen für die Gesunderhaltung, Krankheitsbehandlung und menschenwürdige Sterbebegleitung wieder herausrücken sollen. Genau dafür brauchen wir Versorgungsforschung.

\section{》) Die Versorgungsforschung muss gemeinsam entwickelt werden}

Also, stellen wir uns vom Kopf wieder auf die Füße und lesen mit Spannung und Interesse, wie unsere kompetenten Autoren dieses zukunftswichtige Thema interpretieren:

Gerd Glaeske, prominenter akademischer Versorgungsforscher und Gesundheitsökonom an der Universität Bremen, zeigt beispielhaft die Relevanz der Versorgungsforschung auch für die Urologie auf. Johannes Bruns, Generalsekretär der Deutschen Krebsgesellschaft, berichtet mit seinem Koautor Christoph Kowalski über die „Landung“ der Versorgungsforschung in der Onkologie. Jan Herden,
Urologe an der Universitätsklinik Köln, dokumentiert mit seinen Koautoren die neuesten Daten der HAROW-Studie, übrigens eines echten Leuchtturmprojekts der angewandten Versorgungsforschung, an dem beispielhaft klar wird, dass es nicht um Konkurrenz zu anderen prospektiven Studien geht und durch angeblich zu kurze Laufzeiten kentert, sondern die praktische Evidenz der reinen Lehre und Leitlinien aufblättert und somit als ideale begleitende Ergänzung und Benchmarkinstrument, z. B. für PREFERE, dient. Mit der nicht unumstrittenen Mindestmengenproblematik beschäftigt sich Johannes Huber, Urologe an der Universitätsklinik Dresden. Alwin Weber, Urologe in Michelstadt, greift in den unabdingbaren Zauberkasten der Dokumentationssysteme und Björn Volkmer, urologischer Klinikdirektor in Kassel, belegt an dem ganz praxisnahen Beispiel der Harnsteinbehandlung, wie konkret Therapiepfade durch Versorgungsforschung beeinflusst werden.

Alle Autoren wünschen Ihnen gute Erkenntnisse und nehmen Ihnen die Sorge: Wir beginnen gerade und möchten mit
Ihnen gemeinsam die Versorgungsforschung dahin entwickeln, dass wir Fahrer entscheiden, welches Auto für uns von wem gebaut wird, damit wir das Rennen gewinnen und für unsere Patienten den Siegerpokal entgegennehmen können.

Herzlichst

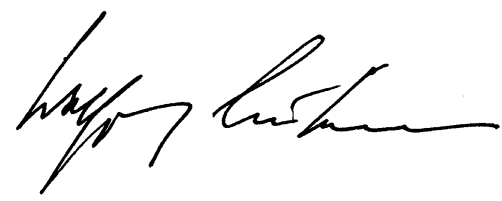

W. Bühmann

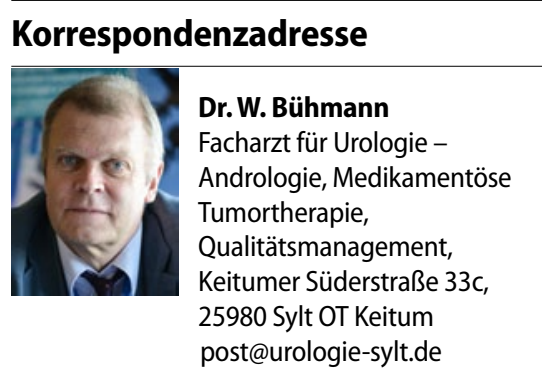

Interessenkonflikt. W. Bühmann gibt an, dass kein Interessenkonflikt besteht.

\section{e.Curriculum Geriatrie: Modul 4 ist online!}

Vertiefen Sie in der Springer Medizin e.Akademie Ihr geriatrisches Fachwissen.

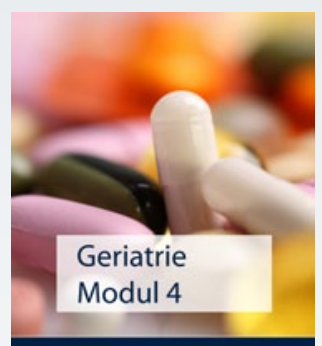

Das Modul 4

„Polymedikation im Alter"

ist:

- mit 5 CME-Punkten zertifiziert,

- interaktiv und multimedial,

- praxisnah mit zahlreichen

Fallbeispielen und Empfehlungen, Ein Kooperationsprojekt mit:

- kostenfrei!
Punkten Sie jetzt kostenfrei in der e.Akademie!

Sie finden diese und weitere Fortbildungsmodule im

e.Curriculum Geriatrie unter:

www.springermedizin.de/ecurriculum-geriatrie

Robert Bosch Stiftung
๑DGG Deutsche Gesellsch
ür Geriatrie e.V. 